\section{Fracture diagnosis and assessment}

\section{Stephen Clarke}

Whilst thorough orthopaedic assessment will identify the bone(s) that have been injured, diagnostic imaging is required to classify fracture, facilitate accurate fracture planning and allow for an estimation of costs and prognosis. Sedation or general anaesthesia will be required to facilitate diagnostic imaging and the onus is on the attending clinician to be sure that the traumatized patient has been adequately stabilized beforehand.

Radiography is the most commonly used way of imaging fractures of the appendicular skeleton. A minimum of two high-quality orthogonal radiographs should be obtained, which should include the joints immediately proximal and distal to the fractured bone. For some other regions, for example the carpus and tarsus, oblique and/ or stressed projections can provide additional information. Obtaining radiographic projections of the intact, contralateral bone provides a useful resource for fracture planning. Particular attention should be given to the fracture location and configuration as well as the overall appearance of the bones, and these should be considered along with the history and clinical examination findings, in order to be sure that is no reason, other than trauma, for the development of the fracture.

For certain fractures, advanced imaging will allow superior assessment. Optimizing the information gained may or may not change the eventual management, however it can improve confidence with regards to decision making, aid surgical planning and prognostication. Of the advanced imaging techniques available computed tomography (CT) is the most useful to the fracture surgeon. In the appendicular skeleton CT would be the imaging technique of choice for fractures of the tarsus and carpus; it can also be helpful for assessing pelvic or articular fractures. For fractures of the axial skeleton, CT is invaluable and would be the preferred imaging technique where possible. CT has the added advantage in that it can also allow for rapid prototype modelling for surgical planning and rehearsal, as well as creation of patient-specific three-dimensional printed drill guides and osteotomy/reduction guides.

\section{KEY LEARNING OBJECTIVES}

- Explain the importance of obtaining preoperative radiographs

- Classify fractures based on their location and configuration

- Recognize the situations where advanced imaging may be beneficial

\section{MULTIPLE CHOICE QUESTIONS}

1. What does preoperative radiographic fracture assessment allow the surgeon to do?

(A) Create a fracture management plan

(B) Estimate prognosis for fracture healing and restoration of mobility

(C) Provide an estimate of costs

(D) All of the above

2. Not all fractures are caused by significant trauma. Which of the following would be the LEAST suggestive that a pathological fracture was present?

(A) A comminuted fracture configuration in the absence of significant traumatic injury

(B) Mandibular fracture sustained during chewing a toy in a 15-year-old Yorkshire Terrier with renal disease

(C) Tibial tuberosity avulsion in a Staffordshire Bull Terrier puppy

(D) Folding fracture configuration in an 8-weekold puppy being fed an all-meat diet

3. When assessing a fractured bone, CT can provide superior information than conventional radiography. For which one of these fractures would CT evaluation be of most help?

(A) Y-T fracture of the distal humerus

(B) Acetabular fracture

(C) Comminuted mid-diaphyseal tibial fracture

(D) Capital physeal fracture

\section{External skeletal fixation}

\section{Eithne Comerford}

\section{DEFINITION, PRINCIPLES AND CLASSIFICATION}

External skeletal fixators are devices consisting of:

- Percutaneous transcortical pins
- Clamps

- Connecting bars.
External skeletal fixators are classified according to the number and location of connecting bars used: types I-III and hybrid.

\section{IMPLANTS}

- Pins. These are placed percutaneously and transosseously and are always bicortical. Pins may be smooth or threaded. Threaded pins have improved pull-out resistance and greater longevity than smooth pins

- Clamps. A number of different makes are available (e.g. Securos, Imex, Veterinary Instrumentation Kirschner Ehmer style). Clamps join pins to the connecting bar(s). Acrylic and epoxy resin can be 


\section{Orthopaedics for beginners}

used to fix pins to connecting bars and can be connecting bars as well

- Connecting bars. These can be made of steel, carbon fibre composite, aluminium, titanium, epoxy resin/acrylic or thermoplastic. They unite the pins and transfer force across the site of injury to reduce forces at the fracture site.

\section{INDICATIONS FOR ESF}

- Distal limb long bone fractures and proximal limb fractures in cats

- Non-reconstructible fractures

- Open/infected fractures

- Mandibular/maxillary fractures

- Growth deformities

- Protection of articular/periarticular injury

- Vertebral column - stabilization and fractures

- Bone defects

- Shearing injuries and protection of periarticular soft tissue reconstruction.

\section{PRACTICAL APPLICATION OF AN EXTERNAL SKELETAL FIXATOR FOR LONG BONE FRACTURE REPAIR}

1. The limb is normally suspended (toes up), with the animal's weight lifted

2. The fracture is aligned by patient positioning and sterile technique is used. Open reduction for simple fractures. Alignment only for comminuted (non-reconstructible) fractures

3. Pins are inserted through stab incisions, using blunt dissection to reach the bone safely

4. A pilot hole is drilled prior to placement of each ESF pin

5. ESF pins are placed using power tools at slow speed (<150 rpm) to avoid thermal necrosis

6. The first two pins are placed perpendicular to and close to the joints. Pins should be no more than $25 \%$ of bone diameter

7. The connecting bars are assembled on the pins

8. Remaining pins are placed, ensuring to put the clamps on the connecting bar first and to place the drill and the pin through the clamp, or otherwise the pins will not join into the frame

9. All clamps are tightened

10. Postoperative radiographs and frame adjusted if needed, and recheck/tighten clamps

11. Padded dressing for entire limb including foot for 24 hours.

\begin{abstract}
Aftercare
A care sheet for clients is helpful, detailing frame management, strict rest and when to be worried! Normal healing is associated with a serum seal forming around pin sites and does not need cleaning.
\end{abstract}

\section{COMPLICATIONS}

- Construct:

- Pin loosening. This is the most common complication

- Pin bending or breakage - inadequate pin size selected

Soft tissue impingement by external skeletal fixator latrogenic fracture

- External skeletal fixator removal by patient.

\section{KEY LEARNING OBJECTIVES}

- Understand the most common implants as well as practical tips and tricks used in the successful placement of external skeletal fixation (ESF)

- Understand the indications and contraindications for placing ESF to ensure successful fracture healing

- Learn how to manage ESF in the postoperative period and any complications encountered

\section{MULTIPLE CHOICE QUESTIONS}

1. What are external skeletal fixators comprised of?

(A) Transosseous pins, clamps and connecting bars

(B) Intraosseous pins, clamps and connecting bars

(C) Transcutaneous pins, clamps and connecting bars

(D) Circular pins, clamps and connecting bars

2. What is the most common complication encountered with ESF placement?
(A) Pin bending
(B) External skeletal fixator removal
(C) Fissure fractures
(D) Pin loosening

3. How does fracture healing occur with an external skeletal fixator used as the fixation device?
(A) Primary bone healing
(B) Secondary bone healing
(C) Hypertrophic healing
(D) Atrophic healing 